\title{
In Silico Interactions Between Curcumin Derivatives and Monoamine Oxidase-A Enzyme
}

\author{
Mahmoud Mirzaei ${ }^{1,2, *}$ (D) , Hamidreza Nazemi ${ }^{3(\mathbb{D})}$ \\ 1 Bioinformatics Research Center, School of Pharmacy and Pharmaceutical Sciences, Isfahan University of Medical \\ Sciences, Isfahan, Iran; mdmirzaei@ pharm.mui.ac.ir (M.M.); \\ 2 Biosensor Research Center, School of Advanced Technologies in Medicine, Isfahan University of Medical Sciences, \\ Isfahan, Iran; mdmirzaei@ pharm.mui.ac.ir (M.M.); \\ 3 Isfahan Pharmacy Students' Research Committee, School of Pharmacy and Pharmaceutical Sciences, Isfahan University \\ of Medical Sciences, Isfahan, Iran; hamidrezanazemi@ gmail.com (H.N.); \\ * Correspondence: mdmirzaei@ pharm.mui.ac.ir (M.M.);
}

Scopus Author ID 13204227300

Received: 27.04.2021; Revised: 5.06.2021; Accepted: 9.06.2021; Published: 13.08.2021

\begin{abstract}
Curcumin (CUR), as a natural product, has been used in food and traditional medicine for a long time. Based on earlier works, it was supposed that CUR could work to medicate cancer and depression. To this aim, derivatives of CUR were investigated in this work to find a better ligand for potent inhibition of monoamine oxidase-A (MAOA) enzyme, which could cause cancer and depression.3D molecular models of thirty derivatives in addition to the original CUR were obtained from the structural bank, and they were prepared for starting ligands by density functional theory (DFT) calculations. In the next step, the 3D structure of MAOA was obtained and prepared for Molecular Docking (MD) simulations of ligand...target complexes. The results indicated different chemistry features for the investigated ligands, and their corresponding interacting complexes were also different. Quantitative and qualitative results were analyzed to find the best ligand for the most potent interaction with the target. C01 was seen as the best ligand among all investigated derivatives and the original CUR for potent inhibition of MAOA enzyme. Both binding energy and interacting with the co-factor of the enzyme were suitable for this compound.
\end{abstract}

Keywords: cancer; depression; curcumin; monoamine oxidase-A; in silico.

(C) 2021 by the authors. This article is an open-access article distributed under the terms and conditions of the Creative Commons Attribution (CC BY) license (https://creativecommons.org/licenses/by/4.0/).

\section{Introduction}

Very well-known curcumin (CUR) (Figure 1) has been used especially by the people of eastern countries as a food additive or traditional medicine for a long time [1]. Although the original natural product CUR has been seen as a useful compound, its synthetic derivatives have also been developed to improve the features of this mysterious compound for specific applications [2]. CUR has been mostly supposed to be a complementary compound for pharmacotherapy, but its single-standing medicinal function has been approved by further works [3]. The main advantage of this compound is its natural resource providing it available as a lead compound to be optimized for various investigations such as therapies for several diseases [4]. It has been shown that in several types of cancers like prostate and lung cancers, monoamine oxidase-A (MAOA) would be overexpressed to increase the destructive functions of cancer [5]. Gross and co-workers [6] indicated that MAOA could yield prostate cancer growth and corresponding metastasis in patients. Liu and co-workers [7] indicated that MAOA may promote non-small cell lung cancer (NSCLC) progression in patients. Moreover, MAOA is an important enzyme yielding the major type of depression in the series of mood disorders 
[8]. Naoi and co-workers [9] indicated that increased expression of MAOA could catabolize more levels of serotonin and norepinephrine to decrease their levels in the brain to yield major depression disorder. When a patient is dealing with cancer therapy, they may lose their hope for living more, making them disappointed people without any hope for being safe and sound anymore [10]. Therefore, several other protocols in the fields of psychology and psychiatry have been developed for prescribing to such patients to recover their hope during the therapy of cancer [11]. When a patient is dealing with major depression disorder, MAOA could be overexpressed, increasing the possibility of the occurrence of cancer [12]. Then, the challenging problem is that depression causes cancer or vice versa, but in both cases, if such MAOA enzyme could be inhibited by a naturally known compound such as CUR, either destructive function of cancer cells or major depression or mood disorder could be solved for the patients dealing with health problems of cancer or depression mood disorder. Within this work, such a hypothesis of MAO inhibition by CUR derivatives was investigated employing the in silico computer-based approach at the molecular scale.

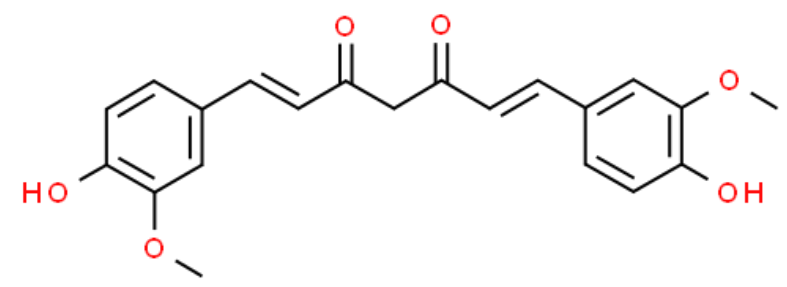

Figure 1. Curcumin (CUR) from ChemSpider (ID: 839564).

Earlier works have introduced various features and functions for CUR compounds, making them useful for possible treatments and therapies in living systems [13]. In cases of both cancer and depression treatments, CUR has been supposed to act its role as a possible direct or in-direct medication [14-17]. In addition to the original CUR, its derivatives have been useful for therapeutic purposes [18]. Although own structural chemistry features of CUR are important, examining its binding affinity against the macromolecular target is also important to determine its potency of interactions in both quantitative and qualitative analyses at the smallest scale [4]. To this aim, in silico approach could provide an environment for careful examining such purpose at the molecular scale to evaluate the interaction properties of ligand...target complexes in binding strength and contributing counterparts [19-22]. Since MAOA includes flavin adenine dinucleotide (FAD) in the active site, a potent ligand needs to interact with FAD to arise inhibiting function [23]. Such details could be very well determined by in silico computer-based approach in Computer-aided drug design and discovery (CADDD) to help solve the complicated problems using molecular-scale analyses [24-26].

\section{Materials and Methods}

Within this work, 3D models of thirty CUR derivatives in addition to its original structure were obtained from the ChemSpider (CS) structural bank [27] with the criteria of existing a chain of seven carbon atoms between two aromatic rings of CUR (Table 1 and Figures 1 and 2). As indicated in Table 1, all obtained structures had a molecular weight (MW) below 500 and partition coefficient (LogP) below 5 referring from CS structural bank [27]. To theoretically validate the starting structures to be considered for in silico investigation, geometries of all obtained 3D models were optimized to reach the minimum energy structures using the B3LYP/3-21G* level of density functional theory (DFT) as implemented in the Gaussian program [28]. In addition to obtaining optimized structures, molecular descriptors 
including dipole moment (DM), energy levels of the highest occupied and the lowest unoccupied molecular orbitals (HOMO and LUMO) were also evaluated for describing chemistry features of the investigated structures (Table 1). After preparing the CUR derivatives for playing ligand roles, 3D model of MAOA enzyme macromolecular structure (ID: 2Z5Y) was obtained from the Protein Data Bank (PDB) [29], and it was prepared for playing the target role of the ligand...target complex formations. Afterward, Molecular Docking (MD) simulation processes were performed to obtain each ligand's best conformation versus the macromolecular target using the AutoDock program [30]. To this aim, a genetic algorithm was referred for 100 runs of conformational search in a $40 * 40 * 40$ grid box ligand...target interactions. By doing MDs processes, quantitative values of binding energy (BE) and inhibition constant (KI) were obtained (Table 1) in addition to qualitative achievements of ligand...target complex formations (Figures 2 and 3). As an advantage of in silico computer-based works, details of complicated systems could be very well detected by doing such computations revealing insightful information for discussing the investigated systems [31-35].

Table 1. Curcumin derivatives (ligands) descriptors*

\begin{tabular}{l|l|l|l|l|l|l|l} 
Ligand & CSID & MW & LogP & DM & HOMO & LUMO & DM \\
\hline C01 & 3635972 & 338 & 3.02 & 3.38 & -5.70 & -2.18 & 3.38 \\
\hline C02 & 5036868 & 368 & 2.92 & 6.43 & -5.30 & -1.87 & 6.43 \\
\hline C03 & 4579941 & 338 & 3.15 & 5.31 & -5.79 & -1.74 & 5.31 \\
\hline C04 & 8672486 & 336 & 4.29 & 2.47 & -6.00 & -2.14 & 2.47 \\
\hline C05 & 3523201 & 308 & 3.29 & 2.56 & -6.11 & -2.24 & 2.56 \\
\hline C06 & 28683119 & 322 & 3.69 & 2.72 & -5.52 & -2.08 & 2.72 \\
\hline C07 & 130011 & 308 & 3.39 & 2.87 & -5.80 & -1.97 & 2.87 \\
\hline C08 & 21427422 & 368 & 2.92 & 4.16 & -5.59 & -2.11 & 4.16 \\
\hline C09 & 26372043 & 368 & 2.82 & 5.46 & -5.93 & -2.00 & 5.46 \\
\hline C10 & 24660343 & 324 & 2.94 & 1.97 & -5.60 & -2.00 & 1.97 \\
\hline C11 & 5042585 & 368 & 2.82 & 3.84 & -5.56 & -1.90 & 3.84 \\
\hline C12 & 2764560 & 354 & 2.62 & 2.72 & -5.66 & -2.03 & 2.72 \\
\hline C13 & 2963780 & 336 & 4.41 & 3.67 & -6.03 & -1.95 & 3.67 \\
\hline C14 & 129416 & 338 & 3.15 & 5.31 & -5.79 & -1.74 & 5.31 \\
\hline C15 & 4949186 & 412 & 4.12 & 4.33 & -4.89 & -4.61 & 4.33 \\
\hline C16 & 8604661 & 370 & 2.16 & 6.10 & -5.43 & -2.04 & 6.10 \\
\hline C17 & 10156030 & 396 & 4.24 & 1.96 & -6.08 & -1.98 & 1.96 \\
\hline C18 & 4742689 & 336 & 4.41 & 3.67 & -6.03 & -1.95 & 3.67 \\
\hline C19 & 346107 & 340 & 2.48 & 2.48 & -5.57 & -1.96 & 2.48 \\
\hline C20 & 4579942 & 340 & 2.48 & 1.62 & -5.57 & -2.00 & 1.62 \\
\hline C21 & 337294 & 336 & 4.35 & 5.54 & -5.69 & -1.90 & 5.54 \\
\hline C22 & 4579943 & 354 & 2.7 & 5.38 & -5.50 & -1.95 & 5.38 \\
\hline C23 & 2782772 & 368 & 2.76 & 5.40 & -5.64 & -2.02 & 5.40 \\
\hline C24 & 8267367 & 391 & 4.12 & 2.99 & -5.48 & -1.89 & 2.99 \\
\hline C25 & 2786 & 368 & 2.92 & 1.79 & -5.69 & -1.90 & 1.79 \\
\hline C26 & 24673409 & 352 & 3.63 & 1.80 & -5.78 & -1.93 & 1.80 \\
\hline C27 & 24676639 & 366 & 4.23 & 2.13 & -5.63 & -1.94 & 2.13 \\
\hline C28 & 4724491 & 368 & 2.76 & 5.32 & -5.65 & -2.05 & 5.32 \\
\hline C29 & 8445961 & 370 & 2.37 & 2.32 & -5.53 & -1.93 & 2.32 \\
\hline C30 & 24663054 & 382 & 2.66 & 7.01 & -5.46 & -1.87 & 7.01 \\
\hline CUR & 839564 & 368 & 2.92 & 1.79 & -5.68 & -1.91 & 1.79 \\
\hline Ch & & & & & & \\
\hline
\end{tabular}

* ChemSpider ID (CSID), Molecular Weight (MW), Partition Coefficient (LogP), Dipole Moment (DM) in Debye, The Highest Occupied Molecular Orbital (HOMO) in eV, The Lowest Unoccupied Molecular Orbital (LUMO) in eV. See Figures 2 and 3 for ligands representations and interaction details. 


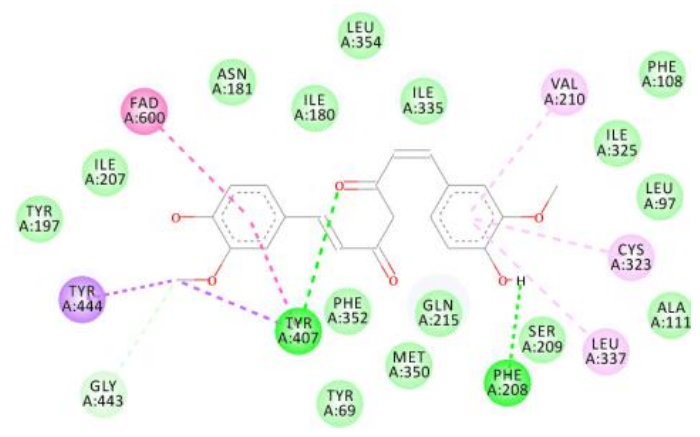

Figure 2. The interacting CUR...MAOA complex.

\section{Results and Discussion}

The original CUR and thirty of its derivatives based on an existing chain of seven carbon atoms between two aromatic rings (Figure 1) were investigated in this work by performing in silico computer-based DFT calculations and MD simulations. The results of molecular descriptors including MW, LogP, DM, HOMO, and LUMO were summarized in Table 1 for all of the investigated ligand structures. These descriptors are important to describe the chemistry features of ligands for possible prediction or interpretation of their corresponding activities. A quick look at the results could show the importance of structure for defining its features. Structural modifications could significantly change such features, as could be seen by comparing the properties of derivatives with the original CUR. The order of ligands from C01 to $\mathrm{C} 03$ was assigned by the obtained values of $\mathrm{BE}$ of ligand...target complex formations meaning that $\mathrm{C} 01$ was in the best condition whereas $\mathrm{C} 30$ was in the worst condition of complex formation with the target. Ligands with higher / lower values of MW than the original CUR (MW=368) were available in Table 1, showing different types of derivations of the original structure. Additionally, values of $\operatorname{LogP}$ could describe the structures with the different tendencies for solubility in oil/water media as one of the important chemistry features for the compounds. Based on the structural modifications, the values of $\log \mathrm{P}$ were changed for the derivatives compared to the original CUR. Furthermore, electric charge distribution balance conditions were also changed for the derivatives, as seen by different DM values. Parallel with such changes, values of HOMO and LUMO also showed significant changes in derivatives. By such different chemistry features for the investigated ligands, it could be expected that their binding affinity for the formation of interacting ligand...target complexes would be changed.

Table 2. Docking results of interacting CUR derivatives...MAOA complexes.

\begin{tabular}{l|l|l|l|l|l|l|l|l|l|l}
$\mathbf{L}$ & $\mathbf{B E}$ & $\mathbf{K I}$ & $\mathbf{L}$ & $\mathbf{B E}$ & $\mathbf{K I}$ & $\mathbf{L}$ & $\mathbf{B E}$ & KI \\
\hline $\mathbf{C 0 1}$ & -9.42 & 0.12 & & $\mathbf{C 1 1}$ & -8.18 & 1.01 & $\mathbf{C 2 1}$ & -7.62 & 2.58 \\
\hline $\mathbf{C 0 2}$ & -9.33 & 0.14 & & $\mathbf{C 1 2}$ & -8.15 & 1.05 & $\mathbf{C 2 2}$ & -7.25 & 4.83 \\
\hline $\mathbf{C 0 3}$ & -9.11 & 0.21 & & $\mathbf{C 1 3}$ & -8.07 & 1.21 & $\mathbf{C 2 3}$ & -6.99 & 7.55 \\
\hline $\mathbf{C 0 4}$ & -8.87 & 0.31 & & $\mathbf{C 1 4}$ & -7.96 & 1.47 & $\mathbf{C 2 4}$ & -6.73 & 11.72 \\
\hline $\mathbf{C 0 5}$ & -8.81 & 0.35 & & $\mathbf{C 1 5}$ & -7.87 & 1.71 & $\mathbf{C 2 5}$ & -6.68 & 12.66 \\
\hline $\mathbf{C 0 6}$ & -8.80 & 0.35 & & $\mathbf{C 1 6}$ & -7.86 & 1.72 & $\mathbf{C 2 6}$ & -6.67 & 13.01 \\
\hline $\mathbf{C 0 7}$ & -8.74 & 0.39 & & $\mathbf{C 1 7}$ & -7.85 & 1.75 & $\mathbf{C 2 7}$ & -6.57 & 15.15 \\
\hline $\mathbf{C 0 8}$ & -8.59 & 0.51 & $\mathbf{C 1 8}$ & -7.79 & 1.96 & $\mathbf{C 2 8}$ & -6.46 & 18.37 \\
\hline C09 & -8.59 & 0.51 & & $\mathbf{C 1 9}$ & -7.77 & 2.02 & $\mathbf{C 2 9}$ & -6.41 & 19.94 \\
\hline C10 & -8.49 & 0.59 & & $\mathbf{C 2 0}$ & -7.75 & 2.07 & $\mathbf{C 3 0}$ & -6.04 & 37.12 \\
\hline CUR & -7.21 & 5.32 & & & & & & & \\
\hline
\end{tabular}

${ }^{*} \mathrm{~L}$ is the ligand in complex with MAOA. The units of BE (Binding Energy) and KI (Inhibition Constant) are $\mathrm{kcal} / \mathrm{mol}$ and $\mathrm{uM}$. See Figures 2 and 3 for interaction details. 


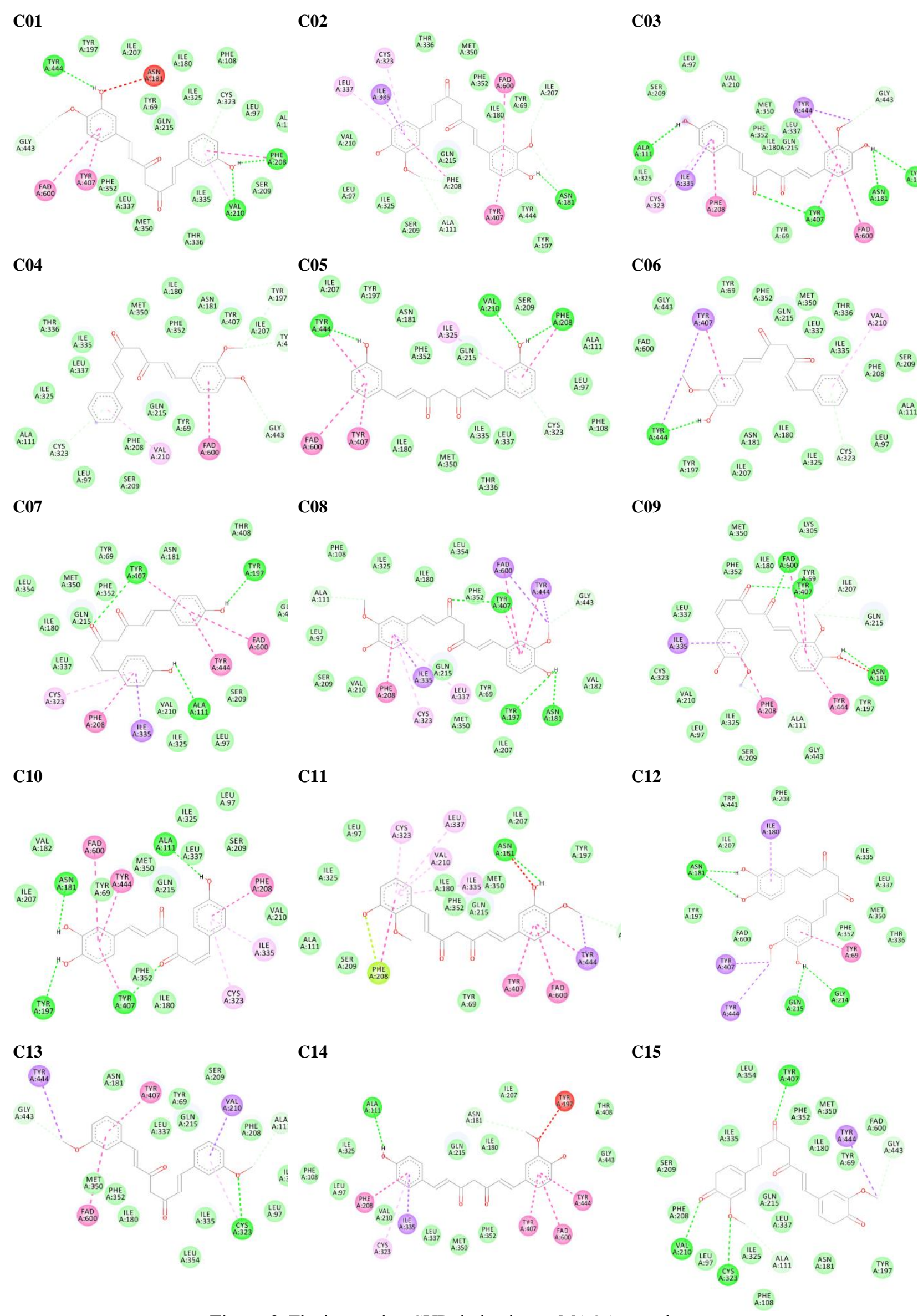

Figure 3. The interacting CUR derivatives...MAOA complexes. 


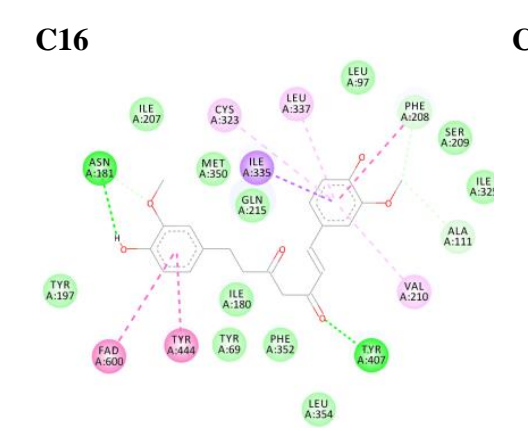

\section{C17}

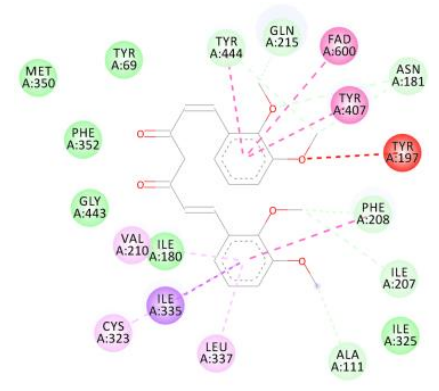

LEU
C18

$\underset{\text { T:R }}{\text { T:197 }}$

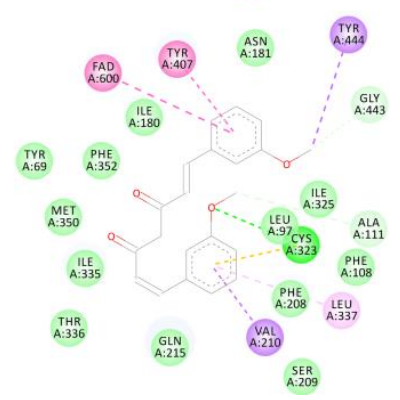

C19

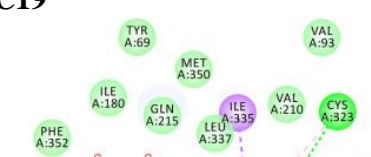

(A.00
C20

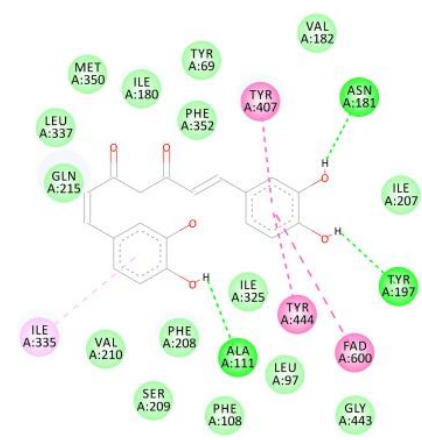

$\mathrm{C22}$

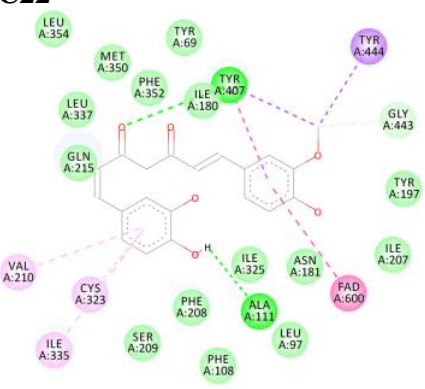

$\mathrm{C23}$

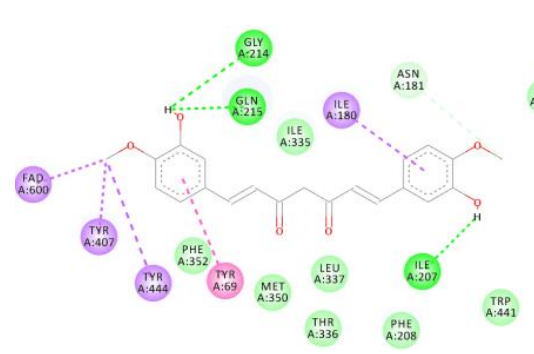

C21

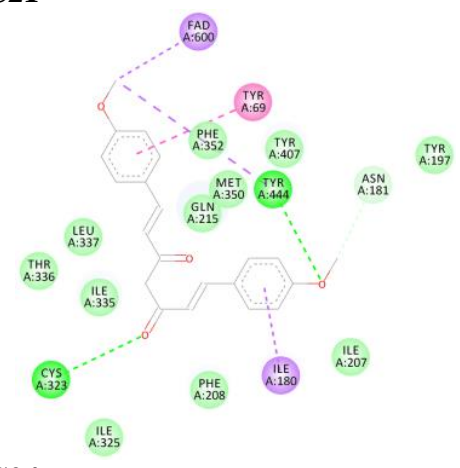

C24

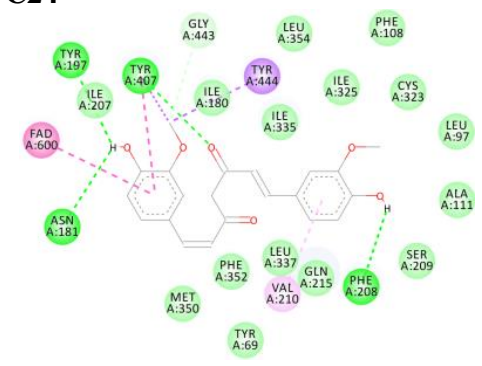

C27
$\mathrm{C} 28$
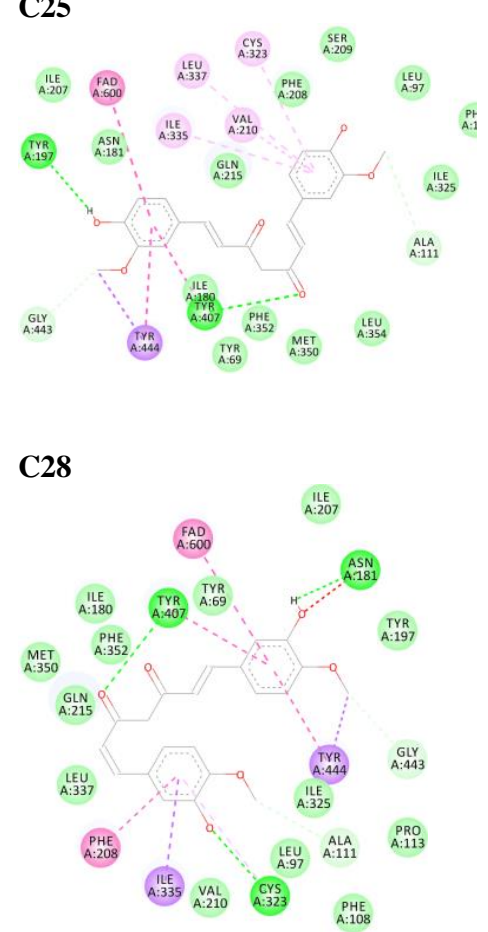

C26

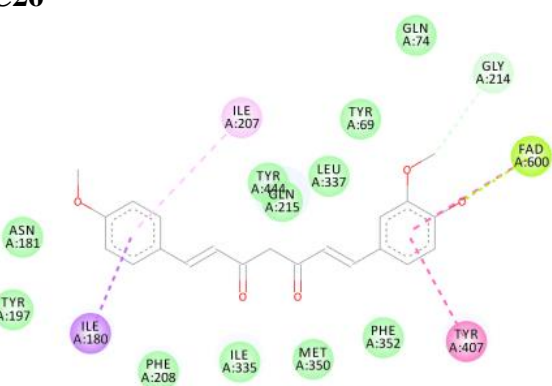

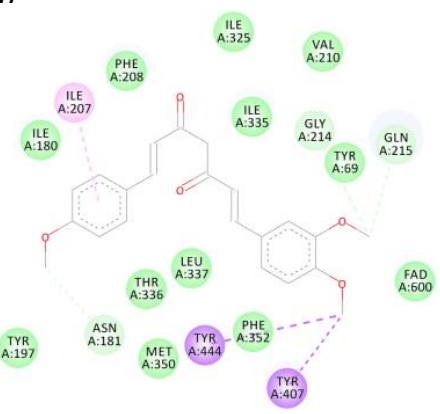

C30
C29

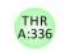

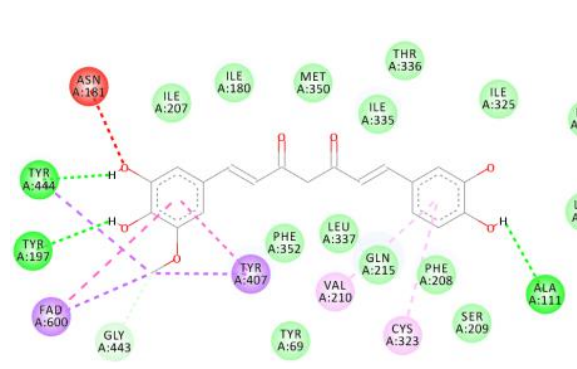

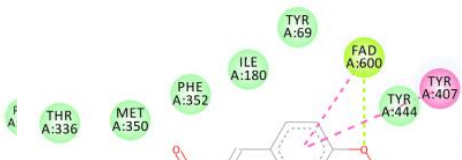

A:335

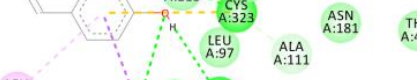

LEU
A:B37

Figure 3 (Continued). The interacting CUR derivatives... MAOA complexes. 
Quickly analyzing the results of Table 2 and Figures 2 and 3 could show such expectations. The values of $\mathrm{BE}$ and $\mathrm{KI}$ were different for complexes and graphical representations of interacting complexes. More specifically, the existence of FAD in the interacting counterparts is an important feature for mentioning about potent interaction of the ligand with the macromolecular target for inhibiting purposes. Such a trend could be seen for some derivatives, especially those with better values of BE and KI for complex formation compared to the original CUR complex. Indeed, FAD plays an important role in the activity of MAOA, and the interaction of a ligand with it to somehow chelate it could help inhibit the activity of the enzyme. To this aim, besides the quantitative analyses of BE and KI, the qualitative analyses could show the advantage of such complex formation to achieve the purpose of enzyme inhibition. The results were more or less different from the original CUR and its corresponding complex, meaning that the lead optimization could yield different results, and careful discussing them for a true choice could be an important task. The results of Figures 2 and 3 for the conformational search of floating ligands versus target could show that the change of spherical shapes of ligand caused by initial structural modification or flexibility of ligand in MD simulation process could yield different conditions for the interacting ligand...target complex formations. Such in silico results could be important in various aspects, from showing the condition of ligand...target interactions until the ligand changes versus the target for the formation of interacting complexes. Based on such obtained results of this work, it was found that the chemistry features of derivatives were different compared to the original CUR in the ligand roles. The results of their corresponding interacting complex formations were also different for such molecular systems. Careful discussion of these in silico results should be done to see the effectiveness of CUR ligands for possible inhibiting of MAOA enzyme from its further destructive activity in the living system. The results of individual ligands in Table 1 and Figure 1 and their corresponding complexes in Table 2 and Figures 2 and 3 would be discussed by following the next part.

Based on the obtained results, some trends could be specifically discussed according to this work's major problem: finding a CUR derivative for potent interaction with MAOA enzyme with inhibitory purpose at the molecular scale. To this aim, thirty CUR derivatives and the original CUR were investigated regarding their individual chemistry features and corresponding binding affinity versus the macromolecular target. As mentioned earlier, overexpression of MAOA could yield destructive activity in the cases of cancer and depression, in which its inhibition could provide more help for the patients. The existence of FAD co-factor in MAOA is important to be noticed, in which it could stimulate the oxidative activity of MAOA, especially in the case of overexpression. Therefore, it is important to somehow chelate FAD to prevent from dealing with MAOA to inhibit enzyme activity. The original CUR showed its ability to interact with FAD in complex formation with MAOA (Figure 2); therefore, it was a good point to investigate more potent inhibitors of this lead compound by structural modifications in derivative compounds. It is worth noting that the structural modification of a lead compound is a way to find a better inhibitor for enzyme inhibition in drug design and discovery methodologies. To do so, modified derivatives of CUR were investigated here for the purpose of more potent inhibition of MAOA activity. The results indicated the importance of types of structural modifications on both individual chemistry features and corresponding binding affinity to the macromolecular target. Energy levels of HOMO and LUMO are important for defining a compound's ability to interact with other structures. As HOMO implies for the level of full electron state possible to work as donor and LUMO implies for the level of 
vacant electron state possible to work as the acceptor of electrons in interactions. Moreover, the energy difference of such HOMO and LUMO states could make it easy to electron transferring or reactivity of such compound. The results showed more proper states of HOMO and LUMO levels of $\mathrm{C} 01$ as an individual ligand compared to the original CUR (Table 1). Its interaction with MAOA was more potent than the original CUR regarding the value of BE. Therefore, C01 was distinguished as the best ligand of CUR derivatives for more potent interaction with the MAOA enzyme. Results of Figure 3 also showed that C01 could interact with FAD, as a dominant co-factor of MAOA for its oxidative activity. By this point, the hypothesis of finding a ligand of CUR for more potent interaction with MAOA enzyme was achieved by analyzing the obtained results in both quantitative and qualitative aspects of this in silico work.

More analyses of the obtained results could show different aspects for assigning the binding strength of ligand...target complexes, such as the number of interactions and their types. In addition to the quantitative values of $\mathrm{BE}$ and $\mathrm{KI}$, the qualitative conditions of interactions could be seen as important for the purpose of potent enzyme inhibition. Both of them together could introduce a ligand for potent interaction with the target, in which the current results indicated $\mathrm{C} 01$ as the most potent one among the investigated ligands. Actually, the initial list of ligands of this work included too many compounds, but they were restricted to thirty ligands according to the obtained qualitative and quantitative results for interacting with MAOA as described here as potent compounds for this purpose.

\section{Conclusions}

This in silico computer-based work was performed to investigate the CUR derivatives for potent inhibition of MAOA enzyme activity. It was mentioned that overexpressed MAOA enzymes could yield cancer and depression for the patients; therefore, finding a potent inhibitor could be very much useful for therapeutic purposes. Among thirty derivatives of investigated CUR, C01 was seen to act as the most potent inhibitor of MAOA with the best value of BE and showing the interaction with FAD co-factor. This is a must that the inhibitor could chelate the FAD co-factor for inhibiting the activity of MAOA. Other ligands, twenty-nine ligands, were seen to be in interaction condition with MAOA but less efficient than $\mathrm{C} 01$ and more or less efficient than the original CUR. As a final concluding remark, structural modification of CUR yielded a better ligand for more potent interaction with MAOA target to probably inhibit its activity.

\section{Funding}

This research received no external funding.

\section{Acknowledgments}

The support of this work by the research council of Isfahan University of Medical Sciences under grant number 297108 is acknowledged.

\section{Conflicts of Interest}

The authors declare no conflict of interest. 


\section{References}

1. Ghaneifar, Z.; Yousefi, Z.; Tajik, F.; Nikfar, B.; Ghalibafan, F.; Abdollahi, E.; Momtazi-Borojeni, A.A. The potential therapeutic effects of curcumin on pregnancy complications: novel insights into reproductive medicine. IUBMB Life 2020, 72, 2572-2583, https://doi.org/10.1002/iub.2399.

2. Noor, H.; Ikram, A.; Rathinavel, T.; Kumarasamy, S.; Nasir Iqbal, M.; Bashir, Z. Immunomodulatory and anti-cytokine therapeutic potential of curcumin and its derivatives for treating COVID-19 - a computational modeling. Journal of Biomolecular Structure and Dynamics 2021, https://doi.org/10.1080/07391102.2021.1873190.

3. Scazzocchio, B.; Minghetti, L.; D’Archivio, M. Interaction between gut microbiota and curcumin: a new key of understanding for the health effects of curcumin. Nutrients 2020, 12, 2499, https://doi.org/10.3390/nu12092499.

4. Mirzaei, M.; Harismah, K.; Soleimani, M.; Mousavi, S. Inhibitory effects of curcumin on aldose reductase and cyclooxygenase-2 enzymes. Journal of Biomolecular Structure and Dynamics 2021, 39, https://doi.org/10.1080/07391102.2020.1800513.

5. Yang, X.; Zhao, D.; Li, Y.; Li, Y.; Cui, W.; Li, Y.; Li, H.; Li, X.; Wang, D. Potential monoamine oxidase A inhibitor suppressing paclitaxel-resistant non-small cell lung cancer metastasis and growth. Thoracic Cancer 2020, 11, 2858-2866, https://doi.org/10.1111/1759-7714.13617.

6. Gross, M.E.; Agus, D.B.; Dorff, T.B.; Pinski, J.K.; Quinn, D.I.; Castellanos, O.; Gilmore, P.; Shih, J.C. Phase 2 trial of monoamine oxidase inhibitor phenelzine in biochemical recurrent prostate cancer. Prostate Cancer and Prostatic Diseases 2021, 24, 61-68, https://doi.org/10.1038/s41391-020-0211-9.

7. Liu, F.; Hu, L.; Ma, Y.; Huang, B.; Xiu, Z.; Zhang, P.; Zhou, K.; Tang, X. Increased expression of monoamine oxidase $\mathrm{A}$ is associated with epithelial to mesenchymal transition and clinicopathological features in nonsmall cell lung cancer. Oncology Letters 2018, 15, 3245-3251, https://doi.org/10.3892/ol.2017.7683.

8. Meyer, J.H.; Ginovart, N.; Boovariwala, A.; Sagrati, S.; Hussey, D.; Garcia, A.; Young, T.; Praschak-Rieder, N.; Wilson, A.A.; Houle, S. Elevated monoamine oxidase a levels in the brain: an explanation for the monoamine imbalance of major depression. Archives of General Psychiatry 2006, 63, 1209-1216, https://doi.org/10.1001/archpsyc.63.11.1209.

9. Naoi, M.; Maruyama, W.; Shamoto-Nagai, M. Type A monoamine oxidase and serotonin are coordinately involved in depressive disorders: from neurotransmitter imbalance to impaired neurogenesis. Journal of Neural Transmission 2018, 125, 53-66, https://doi.org/10.1007/s00702-017-1709-8.

10. So, W.K.; Marsh, G.; Ling, W.M.; Leung, F.Y.; Lo, J.C.; Yeung, M.; Li, G.K. Anxiety, depression and quality of life among Chinese breast cancer patients during adjuvant therapy. European Journal of Oncology Nursing 2010, 14, 17-22, https://doi.org/10.1016/j.ejon.2009.07.005.

11. Agin-Liebes, G.I.; Malone, T.; Yalch, M.M.; Mennenga, S.E.; Ponté, K.L.; Guss, J.; Bossis, A.P.; Grigsby, J.; Fischer, S.; Ross, S. Long-term follow-up of psilocybin-assisted psychotherapy for psychiatric and existential distress in patients with life-threatening cancer. Journal of Psychopharmacology 2020, 34, 155166, https://doi.org/10.1177/0269881119897615.

12. Wang, Y.H.; Li, J.Q.; Shi, J.F.; Que, J.Y.; Liu, J.J.; Lappin, J.M.; Leung, J.; Ravindran, A.V.; Chen, W.Q.; Qiao, Y.L.; Shi, J. Depression and anxiety in relation to cancer incidence and mortality: a systematic review and meta-analysis of cohort studies. Molecular Psychiatry 2020, 25, 1487-1499, https://doi.org/10.1038/s41380-019-0595-x.

13. Fallahi, F.; Borran, S.; Ashrafizadeh, M.; Zarrabi, A.; Pourhanifeh, M.H.; Mahabady, M.K.; Sahebkar, A.; Mirzaei, H. Curcumin and inflammatory bowel diseases: From in vitro studies to clinical trials. Molecular Immunology 2021, 130, 20-30, https://doi.org/10.1016/j.molimm.2020.11.016.

14. Harikrishnan, A.; Khanna, S.; Veena, V. Design of new improved curcumin derivatives to multi-targets of cancer and inflammation. Current Drug Targets 2021, 22, 573-589, https://doi.org/10.2174/1389450121666200804113745.

15. Fusar-Poli, L.; Vozza, L.; Gabbiadini, A.; Vanella, A.; Concas, I.; Tinacci, S.; Petralia, A.; Signorelli, M.S.; Aguglia, E. Curcumin for depression: a meta-analysis. Critical reviews in Food Science and Nutrition 2020, 60, 2643-2653, https://doi.org/10.1080/10408398.2019.1653260.

16. Nadjafi, S.; Hosseini, N.; Farhadi, M.; Khojasteh, F. Interaction of curcumin with one subunit of monoamine oxidase-B in comparison with safinamide: an in silico study. Thrita 2019, 8, e90380, https://doi.org/10.5812/thrita.90380. 
17. Nazemi, H.; Mirzaei, M.; Jafari, E. Antidepressant activity of curcumin by monoamine oxidase-A inhibition. Advanced Journal of Chemistry B 2019, 1, 3-9, https://doi.org/10.33945/SAMI/AJCB.2019.1.2.

18. Oglah, M.K.; Mustafa, Y.F. Curcumin analogs: synthesis and biological activities. Medicinal Chemistry Research 2020, 29, 479-486, https://doi.org/10.1007/s00044-019-02497-0.

19. Mirzaei, M.; Harismah, K.; Da'i, M.; Salarrezaei, E.; Roshandel, Z. Screening efficacy of available HIV protease inhibitors on COVID-19 protease. Journal of Military Medicine 2020, 22, 100-107, http://doi.org/10.30491/JMM.22.2.100.

20. Idris, M.O., Adeniji, S.E., Habib, K.; Adeiza, A.A. Molecular docking of some novel quinoline derivatives as potent inhibitors of human breast cancer cell line. Lab-in-Silico 2021, 2, 30-37, https://doi.org/10.22034/labinsilico21021030.

21. Khalid, H., Hussain, R.; Hafeez, A. Virtual screening of Piperidine based small molecules against COVID19. Lab-in-Silico 2020, 1, 50-55, https://doi.org/10.22034/labinsilico20012050.

22. Harismah, K.; Mirzaei, M. In silico interactions of steviol with monoamine oxidase enzymes. Lab-in-Silico 2020, 1, 3-6, https://doi.org/10.22034/labinsilico20011003.

23. Edmondson, D.E., Binda, C.; Mattevi, A. The FAD binding sites of human monoamine oxidases A and B. Neurotoxicology 2004, 25, 63-72, https://doi.org/10.1016/S0161-813X(03)00114-1.

24. Kriz, K.; Řezáč, J. Benchmarking of semiempirical quantum-mechanical methods on systems relevant to computer-aided drug design. Journal of Chemical Information and Modeling 2020, 60, 1453-1460, https://doi.org/10.1021/acs.jcim.9b01171.

25. Acuna, V.V.; Hopper, R.M.; Yoder, R.J. Computer-aided drug design for the organic chemistry laboratory using accessible molecular modeling tools. Journal of Chemical Education 2020, 97, 760-763, https://doi.org/10.1021/acs.jchemed.9b00592.

26. Mirzaei, M. Making sense the ideas in silico. Lab-in-Silico 2020, 1, 31-32, https://doi.org/10.22034/labinsilico20012031.

27. Pence, HE; Williams, A. ChemSpider: an online chemical information resource. Journal of Chemical Education 2010, 87, 1123-1124, https://doi.org/10.1021/ed100697w.

28. Frisch, M.J.; Trucks, G.W.; Schlegel, H.B.; Scuseria, G.E.; Robb, M.A.; Cheeseman, J.R.; Scalmani, G.; Barone, V.J.I.W. Gaussian 09 program. Gaussian Inc., Wallingford, CT. 2009.

29. Berman. H.M.; Westbrook, J.; Feng, Z.; Gilliland, G.; Bhat, T.N.; Weissig, H.; Shindyalov, I.N.; Bourne, P.E. The protein data bank. Nucleic Acids Research 2000, 28, 235-242, https://doi.org/10.1093/nar/28.1.235.

30. Morris, G.M.; Huey, R.; Lindstrom, W.; Sanner, M.F.; Belew, R.K.; Goodsell, D.S.; Olson, A.J. AutoDock4 and AutoDockTools4: automated docking with selective receptor flexibility. Journal of Computational Chemistry 2009, 30, 2785-2791, https://doi.org/10.1002/jcc.21256.

31. Farahbakhsh, Z.; Zamani, M.R.; Rafienia, M.; Gulseren, O.; Mirzaei M. In silico activity of AS1411 aptamer against nucleolin of cancer cells. Iranian Journal of Blood and Cancer 2020, 12, 95-100.

32. Mirzaei, M.; Hadipour, N.L.; Abolhassani, M.R. Influence of C-doping on the B-11 and N-14 quadrupole coupling constants in boron-nitride nanotubes: a DFT study. Zeitschrift für Naturforschung A 2007, 62, 5660, https://doi.org/10.1515/zna-2007-1-208.

33. Mirzaei, M. Calculation of chemical shielding in C-doped zigzag BN nanotubes. Monatshefte für Chemie 2009, 140, 1275, https://doi.org/10.1007/s00706-009-0195-6.

34. Dhiman, P.; Malik, N.; Khatkar, A. Natural based piperine derivatives as potent monoamine oxidase inhibitors: an in silico ADMET analysis and molecular docking studies. BMC Chemistry 2020, 14, 1-6, https://doi.org/10.1186/s13065-020-0661-0.

35. Mirzaei, M. Science and engineering in silico. Advanced Journal of Science and Engineering 2020, 1, 1-2, https://doi.org/10.22034/AJSE2011001. 